Article

\title{
Real-Time Mode Switching and Beam Scanning of High-Gain OAM Waves Using a 1-Bit Reconfigurable Reflectarray Antenna
}

\author{
Ziyang Wang, Xiaotian Pan, Fan Yang *, Shenheng Xu and Maokun Li
}

The State Key Laboratory on Microwave and Digital Communications, Tsinghua National Laboratory for Information Science and Technology, Department of Electronic Engineering, Tsinghua University, Beijing 100084, China; wangziyang1@tsinghua.edu.cn (Z.W.); pxt16@mails.tsinghua.edu.cn (X.P.); shxu@tsinghua.edu.cn (S.X.); maokunli@tsinghua.edu.cn (M.L.)

* Correspondence: fan_yang@tsinghua.edu.cn; Tel.: +86-10-62781482

Received: 11 November 2020; Accepted: 8 December 2020; Published: 18 December 2020

\begin{abstract}
A reconfigurable electromagnetic surface has been studied to realize the adjustable orbital angular momentum (OAM) beams for real-time wireless communication and dynamic target detection in the future. OAM mode switching realized by many previous designs suffers from low gains without OAM beam scanning. In this article, a 1-bit reconfigurable reflectarray antenna is designed, fabricated, and tested for the real-time control of OAM mode switching and large-angle vortex beam scanning in three-dimensional space. The proposed reflectarray surface is composed of 1-bit electronically reconfigurable cells, and the size is $24 \lambda \times 24 \lambda$ with 2304 units. The reconfigurable element is designed by using a radiation patch loading a PIN diode with effective control of two states, "ON" and "OFF", for the demand of $180^{\circ}$ phase difference. The reflectarray surface can be assigned to a code sequence of 0 or 1 by the Field-Programmable Gate Array (FPGA) in real time. Henceforth, the coding surface can dynamically control the generation of high-gain OAM beams, where only the optimized phase distributions on the surface need to be changed according to demand. To verify the concept, a large-scale reflectarray surface is fabricated and measured with an oblique feed at $15^{\circ}$. Different OAM-carrying phase distributions for different OAM beam states are calculated and tested. The test results show that the OAM mode switching between $l=1$ and $l=2$ is realized, and other variable modes such as $l=3$ or $l=5$ can also be achieved by modifying the phase encoding sequence. Furthermore, the direction of the vortex beams can be accurately controlled with gains over $20 \mathrm{dBi}$, and the large-angle vortex beam scanning is verified. Therefore, all results demonstrate that the proposed 1-bit reconfigurable reflectarray is efficient for the regulation and control of OAM-carrying beams for the demand of real-time dynamic wireless communications in the future.
\end{abstract}

Keywords: 1-bit reconfigurable reflectarray antenna; real-time; OAM mode switching; large-angle vortex beam scanning; Field-Programmable Gate Array (FPGA)

\section{Introduction}

Angular momentum, especially orbital angular momentum (OAM), is currently a subject of great interest in academic research. In essence, electromagnetic (EM) waves can carry both linear momentum and angular momentum [1]. OAM L is a kind of angular momentum, and another kind of angular momentum is called spin angular momentum (SAM) S [2], which can be traced as far back as 1909. Moreover, there are three different values of $S$ corresponding to three different polarization states: when S equals 1, it represents the left-handed circularly polarized (LHCP) state; when S equals -1 , it represents the right-handed circularly polarized (RHCP) state; when S equals 0 , it will degenerate to a linear 
polarized (LP) state. OAM is determined by the azimuthal phase term exp(i $\ell \theta)$ of the helical wave-front, in which $\ell$ can take any integer values as $-2,-1$ and +1 , and $\theta$ is the azimuthal angle with a range of 0 to $2 \pi$. In addition, the different OAM modes $\ell$ are orthogonal to each other, which make it possible for mode multiplexing. EM waves carrying OAM are called vortex waves and have aroused great interest iny scholars. They first came to the forefront in optical fields [3], and many novel applications [4-10] have emerged, such as superresolution imaging [4], optical microscopy, and OAM communication [5]. Owing to its novel property, OAM has been widely researched in the microwave field [11-13] for the potential application to increase channel capacity, spectral efficiency, and radar detection. The earliest simulated OAM design was performed in 2007 [11]. Later, an experiment proved the feasibility of OAM realization [12], and a landmark experiment [13] of OAM-based wireless transmission was reported in 2012, which proved that the capacity of communication could be improved over a distance of $442 \mathrm{~m}$ with a fixed bandwidth. Due to the broad prospects of OAM, it is necessary to explore effective methods to realize OAM. Recently, the generation of OAM-carrying beams mainly focused on the helical phase plate [14], spiral parabolic antenna [15], circular-phased array antenna [16,17], and dielectric resonator antenna [18]. Other relatively new reports show that dual-mode OAM states can be generated at different working frequencies, in [19], and that a water-immersed rectangular horn antenna array was fabricated for generating underwater OAM waves [20].

The artificial electromagnetic surface opened a new way to generate OAM, since its flexible regulation functions on an electromagnetic wave. A reflectarray surface is utilized to generate an OAM beam [21]; then, dual polarizations [22] and dual bands [23] for an OAM wave are realized. There are other representative papers on the realization of OAM-carrying beams based on a reflective metasurface [24-26]. A transmitarray surface is also introduced to generate a high-performance OAM wave with $l=1$ [27], since it is without feed blockage, which can improve the efficiencies of a surface antenna. In addition, an anisotropic tensor impedance surface [28] is applied to the generation of OAM. However, the OAM beams generated by period surfaces [29] mentioned above are fixed, and the element is not reconfigurable. Then, the reconfigurable reflectarray surface provides a powerful basis [30-35] for the real-time dynamic adjustment of OAM by changing the coding sequences, and it can be considered in future applications of the vortex imaging system, high throughput communication, and multi-target detection. For the adjustable design of OAM beams by using the reconfigurable surface, there are a few reports [36-38] about the dynamic modulation of the OAM mode. In [36], a digital OAM generator is presented by loading the PIN diodes on the element, and OAM beams with the modes of $l=1$ and $l=2$ are achieved. Thereafter, the 2-bit reconfigurable reflective surface is fabricated to generate multimode OAM beams [37]. A transmissive programmable metasurface [38] is also used to generate a high-efficiency OAM beam, since there are no feed blockages and the realized OAM mode has been presented from $l=-2$ to $l=+2$. Only the adjustable function of the OAM mode are realized by the reflective/transmissive metasurface antennas mentioned above; however, the gains of these metasurface antennas are not high and the OAM beams scanning are not mentioned.

In this article, a 1-bit reconfigurable reflectarray antenna consisting of $48 \times 48$ elements is utilized to dynamically control the mode switching and large-angle scanning of high-gain OAM beams in a 3D space domain. Various OAM-generation phase distributions with an oblique feed are presented. The mode switching between $l=1$ and $l=2$ has been achieved. A large-angle vortex beam scanning approximately $75^{\circ}$ is also realized. Therefore, it is efficient for the arbitrary modulation and control of the OAM-carrying beam due to the proposed large-scale reconfigurable reflectarray surface. It may become a potential candidate for real-time dynamically OAM-based wireless communications in the future. 


\section{Design and Analysis}

\subsection{Design of a Reflectarray Surface for OAM}

A reflectarray antenna is presented in Figure 1 to illustrate the design and scan of OAM beams using 1-bit elements on the surface. The desired compensation phase to generate OAM beams can be calculated by Equation (1) for different OAM modes, where $\mathrm{k}$ is the propagation constant, $\mathrm{rmm}$ is the path length from the feed to different elements on the surface, rmn is the position of the mnth element, $l$ is the mode number of the OAM, $\mathrm{u} 0$ is the desired main-beam direction of OAM, and Фmn is the azimuthal angle for the mnth element. Next, the phase distribution of a 1-bit surface can be calculated by Equation (2) based on the 1-bit phase quantization. Considering that the surface consists of $\mathrm{M} \times \mathrm{N}$ cells illuminated by the feed, the scattered fields from the surface in arbitrary directions can be calculated by Equation (3), where Amn is the amplitude excited on each cells, which is computed by Equation (4). In Equation (4), FP is the feed pattern described by the cos-mode, EP is the element pattern, and $\Gamma \mathrm{mn}$ is the loss of elements on the surface.

$$
\begin{gathered}
\varphi(m, n)_{O A M}=k \times r_{f m n}-k \times r_{m n} \times u_{0}+\Delta \varphi+l \times \Phi_{m n} \\
l=0, \pm 1, \pm 2, \ldots \\
\varphi_{1 b i t O A M}=\left\{\begin{array}{c}
0,-\frac{\pi}{2}<\varphi(m, n)_{O A M}<\frac{\pi}{2} a n d \varphi(m, n)_{O A M}= \pm \frac{\pi}{2} \\
\pi,-\pi<\varphi(m, n)_{O A M}<-\frac{\pi}{2} a n d \frac{\pi}{2}<\varphi(m, n)_{O A M}<\pi
\end{array}\right. \\
E(\theta, \varphi)=\cos \theta \sum_{m=1}^{M} \sum_{n=1}^{N} A_{m n} e^{\left(-j k r_{f m n}+j \varphi_{1 b i t O A M}+j k r_{m n} u\right)} \\
A_{m n}=\frac{F P \times E P \times\left|\Gamma_{m n}\right|}{r_{f m n}}
\end{gathered}
$$

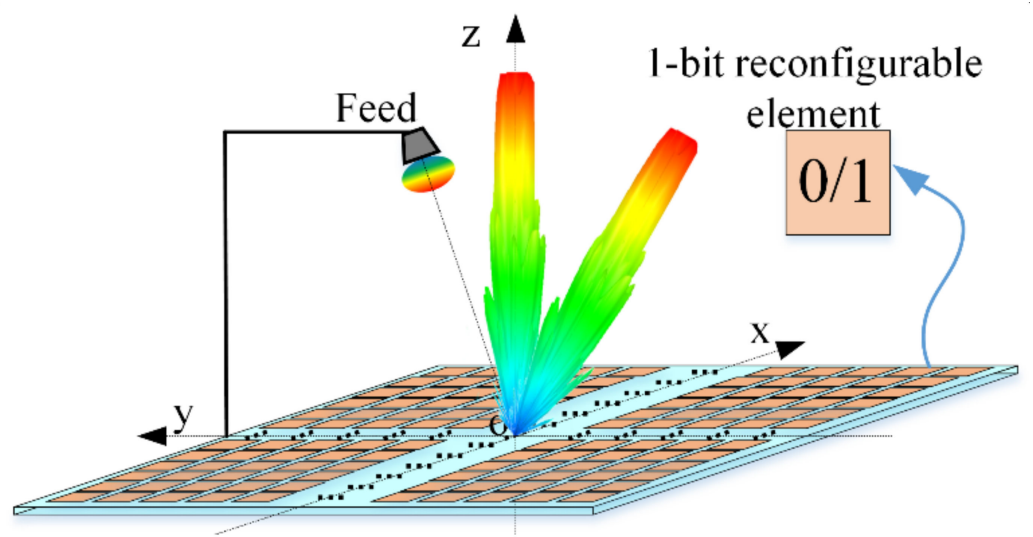

Figure 1. Schematic diagram of a reflectarray antenna for orbital angular momentum (OAM).

\subsection{Configuration and Responses of the 1-Bit Element}

A reconfigurable patch element is designed by loading a PIN diode (Skyworks SMP1340), and the detailed geometrical structure is presented in Figure 2a. The parameters of the 1-bit element are shown as follows: $\mathrm{px}=6.2 \mathrm{~mm}$; $\mathrm{py}=6.0 \mathrm{~mm}$; the thickness of the Taconic TLX-8 (dielectric constant 2.55, loss of tangent 0.0019 ) and FR4 substrates (dielectric constant 4.4, loss of tangent 0.02 ) are $1.58 \mathrm{~mm}$ and $0.5 \mathrm{~mm}$, respectively. The state of the PIN diode is controlled by its bias voltages; the PIN diode will remain in the ON state loading with a voltage of $+0.8 \mathrm{~V}$ and OFF state with a voltage of $-12 \mathrm{~V}$. The magnitude and phase responses for different states are shown in Figure $2 b$. The phase responses are $91.1^{\circ}$ and $-92.5^{\circ}$ in the two states of $\mathrm{ON}$ and OFF at $9.37 \mathrm{GHz}$. Therefore, the phase difference is 
close to $180^{\circ}$, which is consistent with the demand of 1-bit operation. Meanwhile, the element losses in both states are acceptable and less than $0.6 \mathrm{~dB}$.

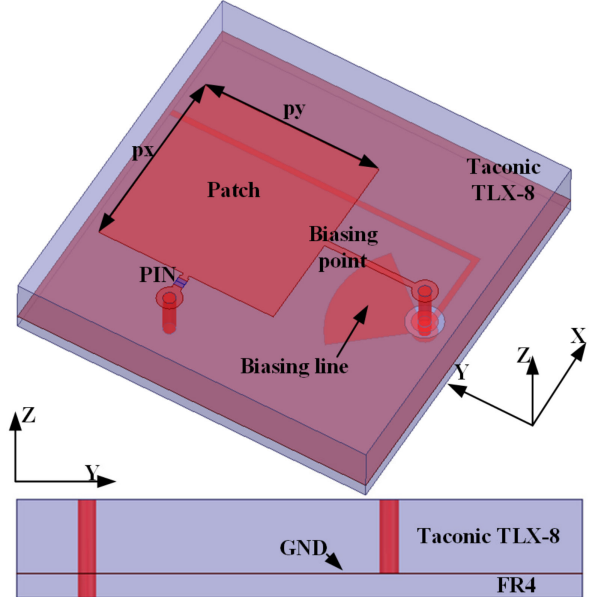

(a)

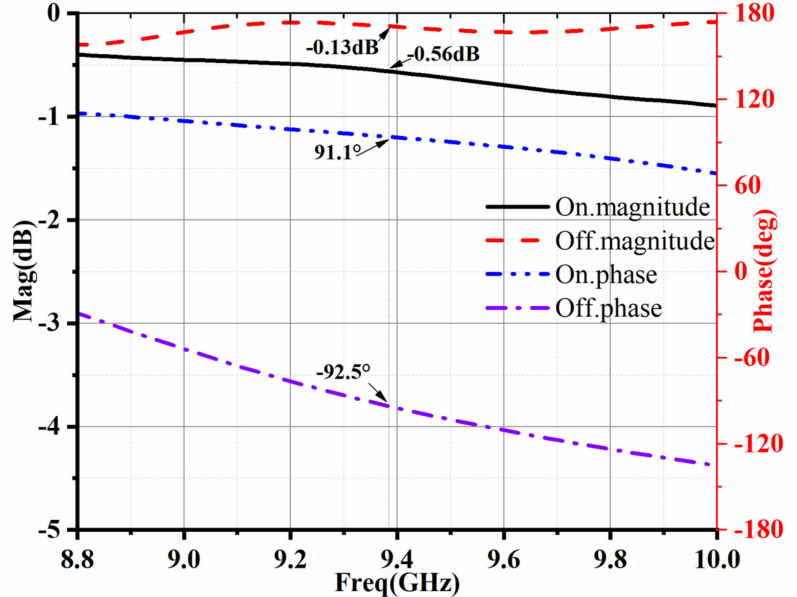

(b)

Figure 2. 1-bit reconfigurable element: (a) geometrical structure; (b) simulated magnitude and phase curves in two states.

\subsection{Realization of Different OAM Beams}

For many published papers, the feed is located above the surface without an oblique angle, where the number of modes can be intuitively observed from the phase distributions. Since the oblique incidence has less feed blockage, different OAM beams with an oblique feed are investigated here. First, the phase distributions with normal incidence for different OAM modes of $l=1$ and $l=2$ are realized in the first and second columns in Figure 3, respectively. The number of OAM modes can be clearly observed from the distributions in the first two columns. Next, the phase distributions with an oblique feed for modes $l=1$ and $l=2$ are also presented in the last two columns. However, the value of OAM modes cannot be directly determined from the phase distributions. In addition, the far-field patterns calculated by Equation (4) are presented in the end line for different cases in Figure 3e-h. The OAM patterns with intermediate null are realized.

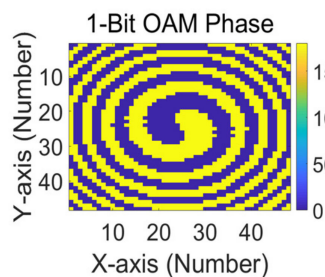

(a)

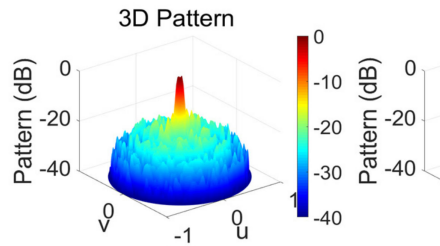

(e)

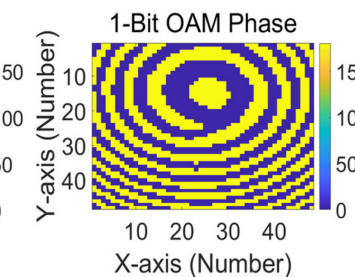

(b)

3D Pattern

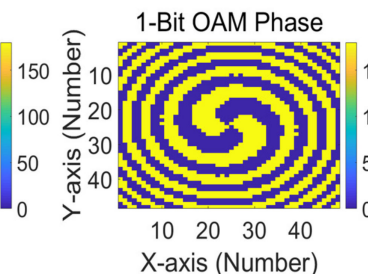

(c)

3D Pattern

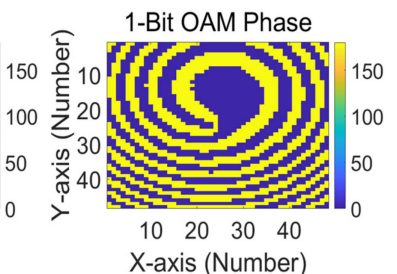

(d)

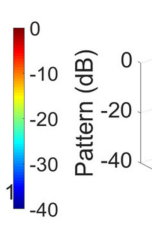

(f)

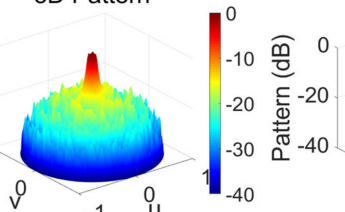

(g)

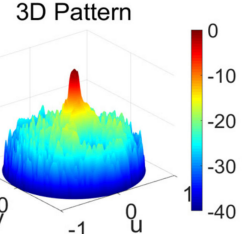

(h)

Figure 3. OAM phase distributions using the 1-bit element: (a) $l=1$ with normal incidence; (b) $l=2$ with normal incidence; (c) $l=1$ with oblique incidence; (d) $l=2$ with oblique incidence. 3D far-field patterns using the 1-bit element: (e) $l=1$ with normal incidence; (f) $l=2$ with normal incidence; (g) $l=1$ with oblique incidence; (h) $l=2$ with oblique incidence. 


\section{Measurement and Discussion}

\subsection{Prototype of the Antenna and Experimental Environment}

A 1-bit reconfigurable reflectarray surface with a size of $24 \lambda \times 24 \lambda$ is fabricated to realize the mode switching and large-angle vortex beam scanning for OAM waves, as shown in Figure $4 \mathrm{a}$. The surface is illuminated by a feed with a $15^{\circ}$ inclination angle and the ratio of focal length and diameter (F/D) of approximately 0.76 , as shown in Figure $4 \mathrm{~b}$. The proposed surface is tested in a microwave near-field chamber, as shown in Figure 4c. The detailed test components are a vector network analyzer, a phase controller, and the proposed antenna with an FPGA control circuit. A standard probe is used to measure the vertical polarization component (y-polar) of the reflected E-filed, where the sampling plane is placed at a distance of $25 \lambda$ away from the reflectarray surface. The probe scans from $-728 \mathrm{~mm}$ to $728 \mathrm{~mm}$ in both $\mathrm{x}$ and $\mathrm{y}$ directions with a sampling period of $16 \mathrm{~mm}$.

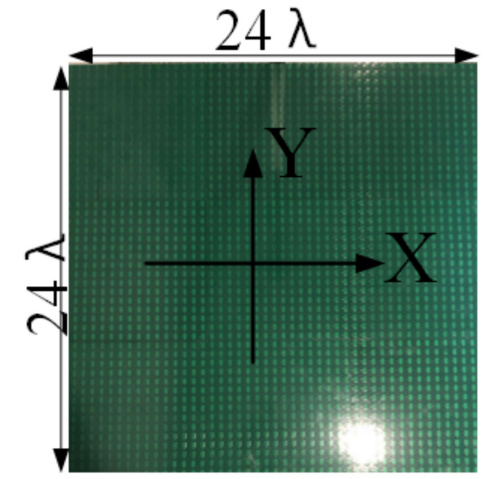

(a)

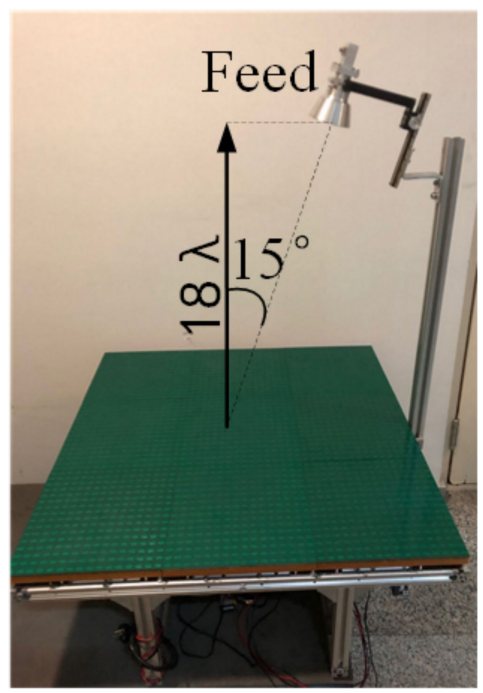

(b)

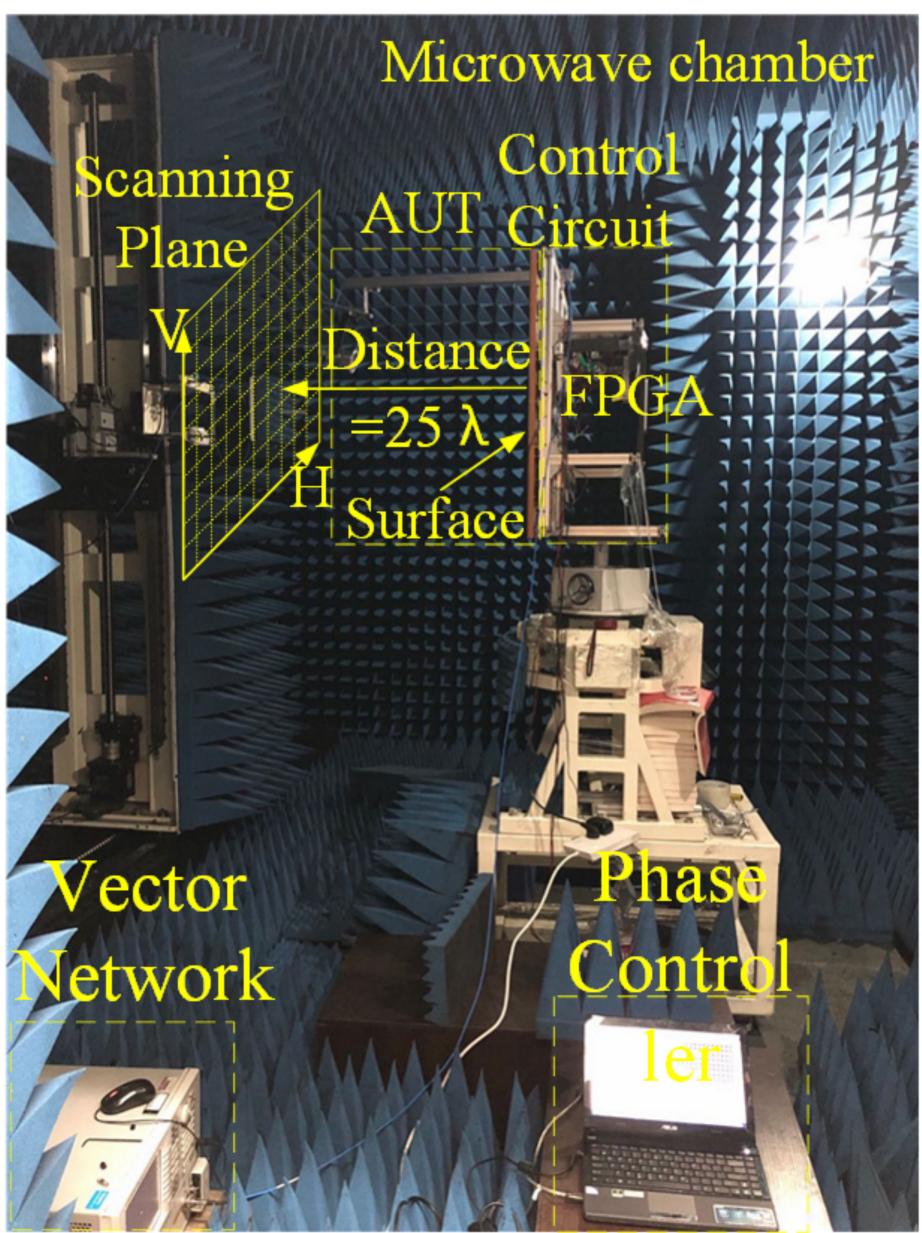

(c)

Figure 4. Antenna prototype and planar near-field scanning microwave chamber: (a) proposed 1-bit surface; (b) prototype of the reconfigurable reflectarray antenna; (c) test components and environment.

\subsection{Mode Switching of the OAM Beam}

In this section, the real-time mode switching of the OAM wave is presented. Due to the advantage of the large-scale reconfigurable surface, high-order modes for OAM carrying can be realized, and the OAM modes can be switched. Then, a typical OAM mode switching between $l=1$ and $l=2$ is validated. By changing the surface control voltages through the FPGA circuit, different surface phase distributions in Figure $3 c, d$ are realized, and the measured near-field data for different modes are displayed in 
Figure 5. The modes can be intuitively identified based on different spiral phase distributions, as shown in Figure 5b,d. The circular-shape magnitude distributions with a null are visible in two cases in Figure 5a,c. Therefore, the feasibility for variable mode generation and switching is confirmed.

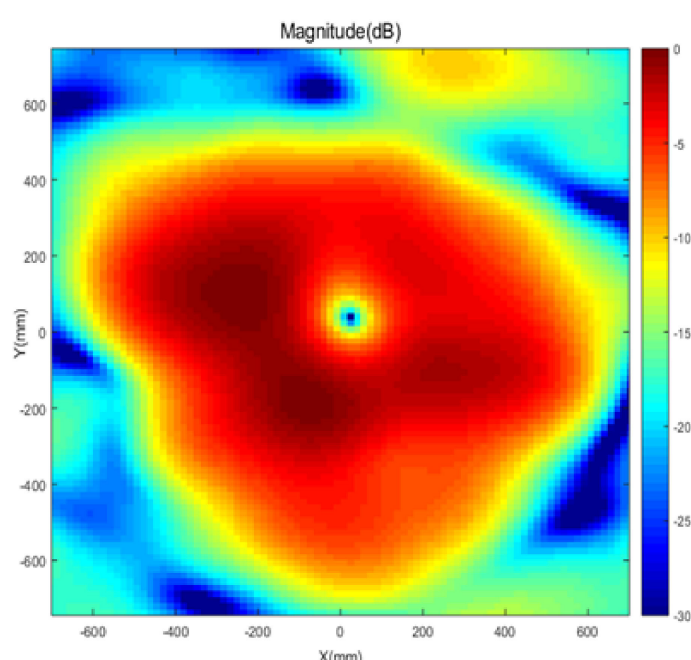

(a)

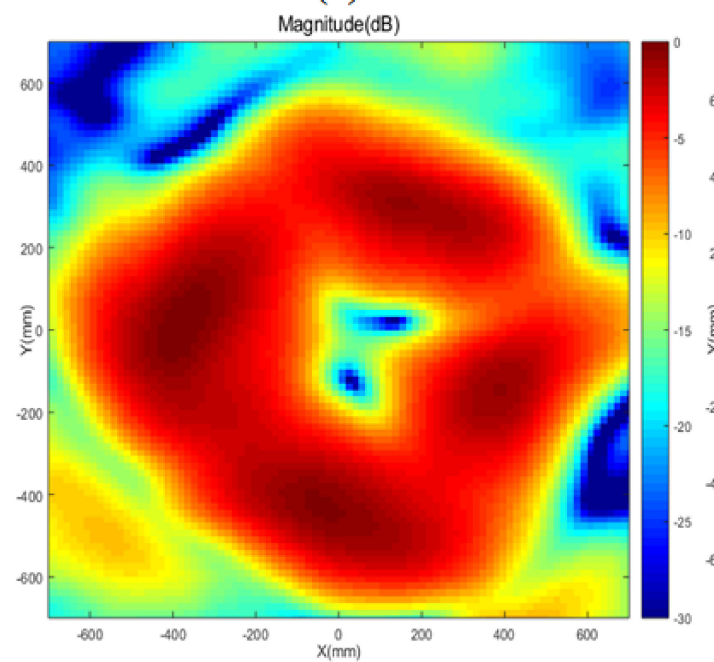

(c)

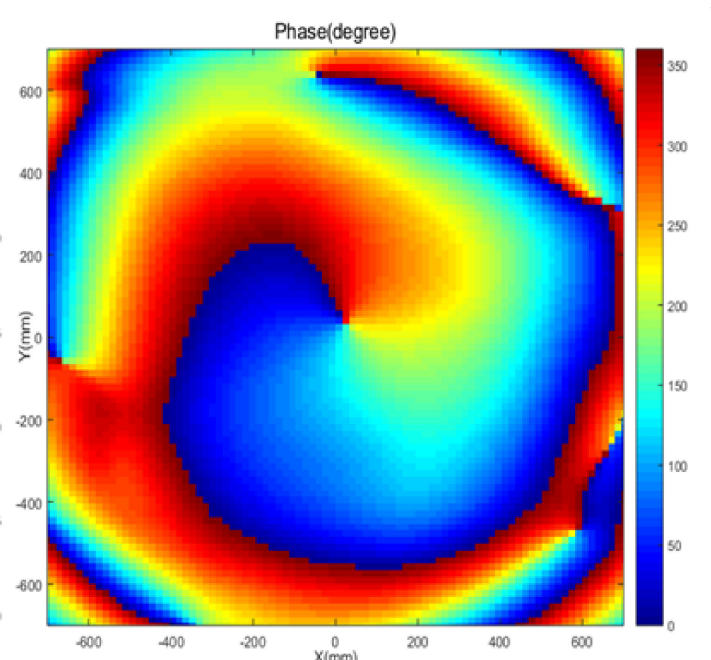

(b)

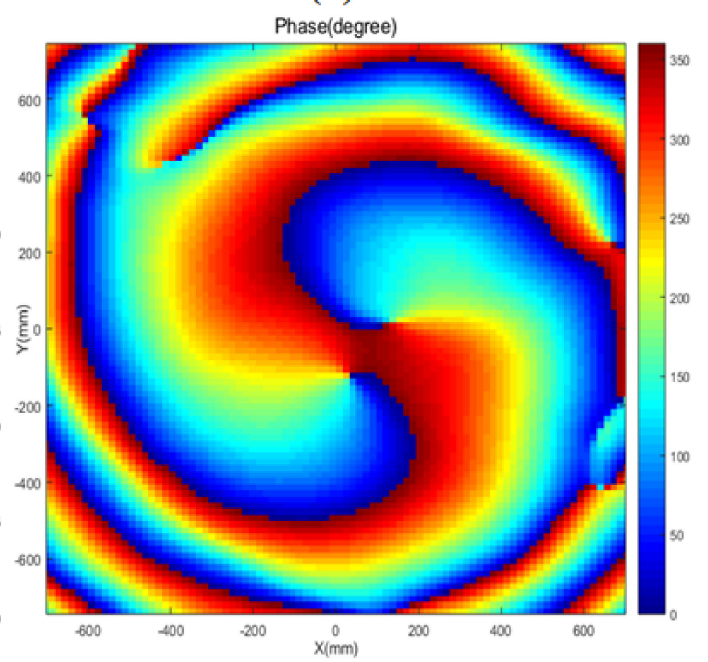

(d)

Figure 5. Measured near-field data of OAM wave: (a) magnitude distribution for $l=1$; (b) phase distribution for $l=1$; (c) magnitude distribution for $l=2$; (d) phase distribution for $l=2$.

Meanwhile, the far-field results of mode switching are presented in Figure 6. In the XOZ and YOZ planes, the test results are consistent with the simulation ones in two different modes, as shown in Figure 6a,b.

Moreover, the measured values of null at $\theta=0^{\circ}$ are lower than $-25 \mathrm{~dB}$ in both modes. The angles of the main lobe for different modes are approximately $2.0^{\circ}$ and $2.8^{\circ}$, respectively, which are narrow among the published papers and will help to increase the distance of OAM-based wireless communications. Next, the measured 3D patterns for mode switching are shown in Figure $6 \mathrm{c}, \mathrm{d}$. In terms of near-field and far-field measured results, the real-time mode switching of the OAM wave is implemented, and the OAM performance is acceptable. 


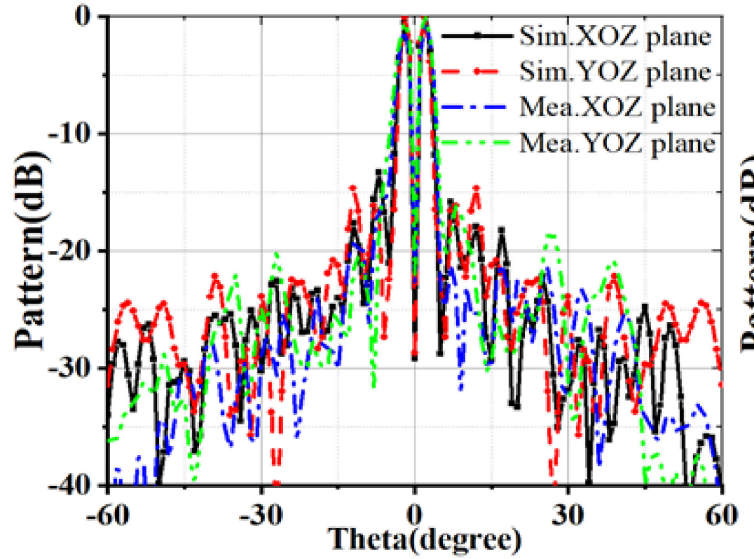

(a)

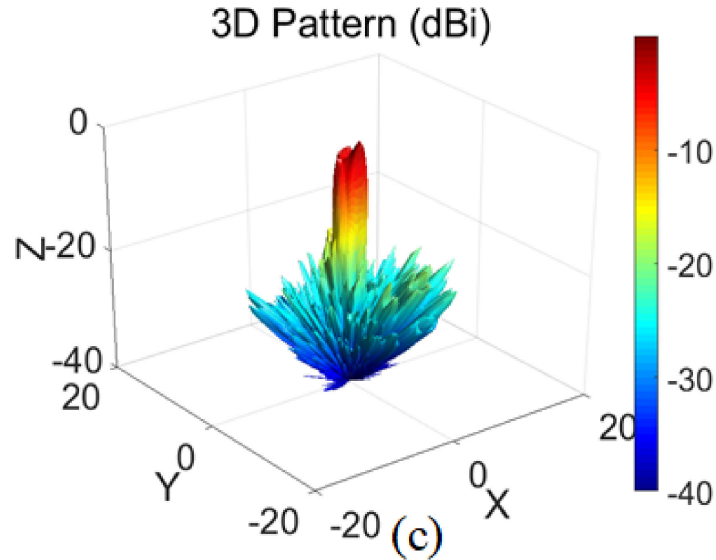

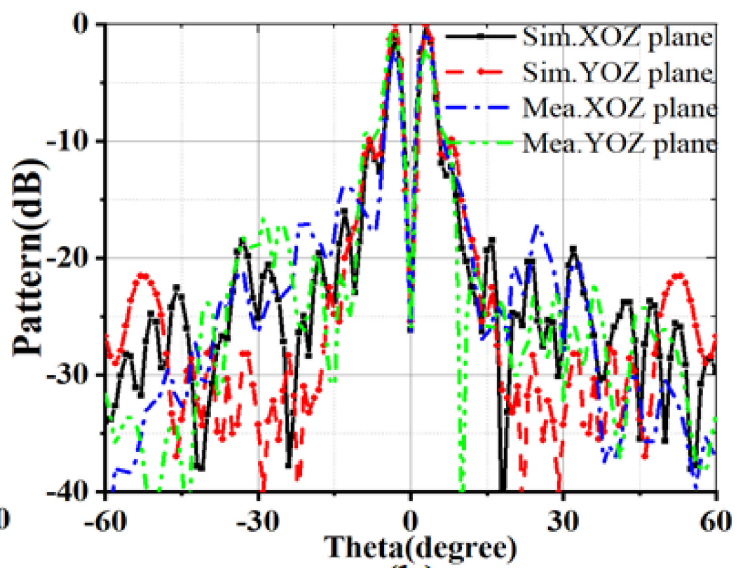

(b)

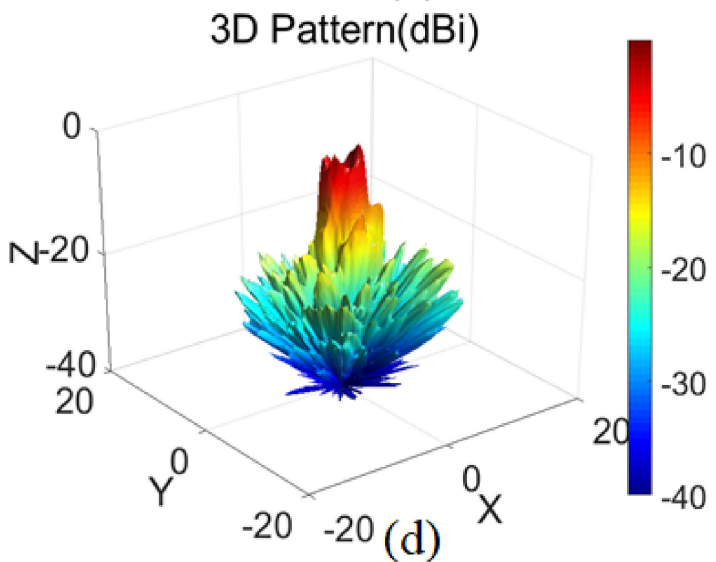

Figure 6. Far-field radiation patterns of OAM: (a) simulated and measured 2D patterns of $l=1$; (b) simulated and measured 2D patterns of $l=2$; (c) measured 3D pattern of $l=1$; (d) measured 3D pattern of $l=2$.

\subsection{Large-Angle Beam Scanning of OAM Wave}

Next, the beam scanning of the OAM wave as a new phenomenon is investigated, which satisfies an important demand for real-time dynamic communications in the future. Some representative far-field measured results of OAM beam scanning in the XOZ plane with mode $l=1$ are presented in Figure 7. Due to the good performance of the 1-bit reconfigurable surface, precision-control of OAM beam scanning is achieved by modifying the surface phase distributions in real time using FPGA for different scanning angles. Moreover, the OAM beam scanning of large angles at $60^{\circ}$ and $75^{\circ}$ are also realized.

The far-field performance of gains and efficiencies for different OAM scanning beams are presented in Table 1. The gains decrease with the increase in scanning beams, as shown in the first line in Table 1.

Table 1. Far-field performance for different orbital angular momentum (OAM) scanned beams.

\begin{tabular}{cccccc}
\hline Parameter & $\mathbf{0}^{\circ}$ & $\mathbf{1 5}^{\circ}$ & $\mathbf{3 0}^{\circ}$ & $\mathbf{4 5}^{\circ}$ & $\mathbf{6 0}^{\circ}$ \\
\hline Gain(dBi) & 27.2 & 26.26 & 25.48 & 23.32 & 20.70 \\
\hline
\end{tabular}

The measured wave-front amplitude and phase distributions corresponding to each OAM scanning beam are presented in Figure 8. The amplitude distributions of a circular shape with a null are realized in all desired directions, as shown in Figure 8a-d. Meanwhile, the phase distributions in counterclockwise rotation with the OAM mode of $l=1$ at different beam scanning angles are perfectly 
displayed as expected in Figure 8e-h. Therefore, by combining the measured near-field and far-field data, the large-angle OAM beams scanning have been achieved.

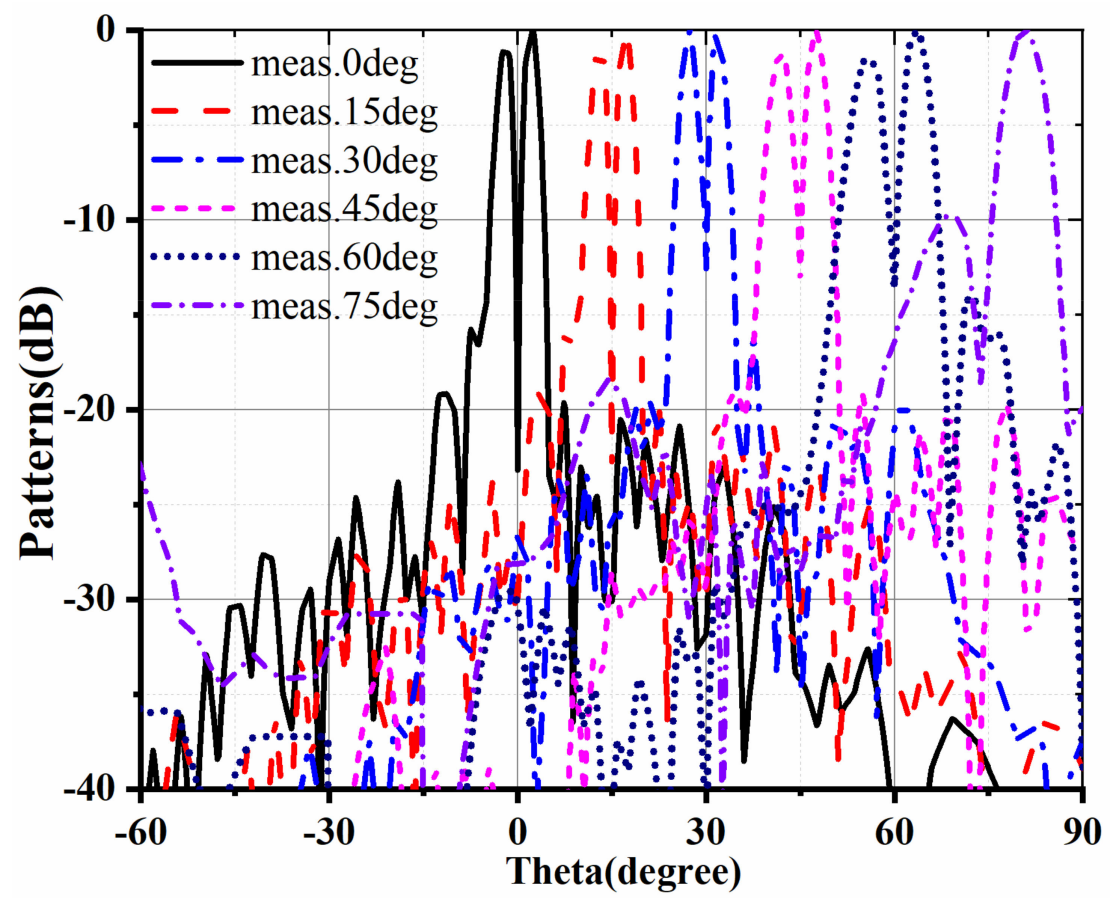

Figure 7. Measured far-field data for beam scanning of OAM in XOZ plane with different angles at $0^{\circ}$, $15^{\circ}, 30^{\circ}, 45^{\circ}, 60^{\circ}, 75^{\circ}$.

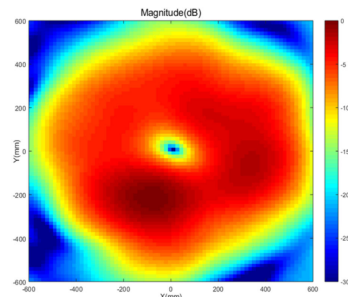

(a)

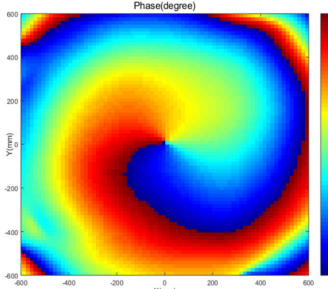

(e)

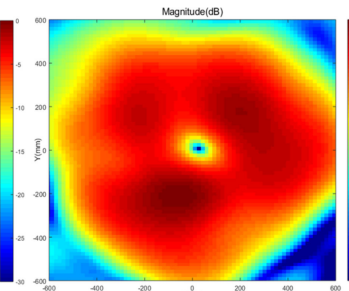

(b)

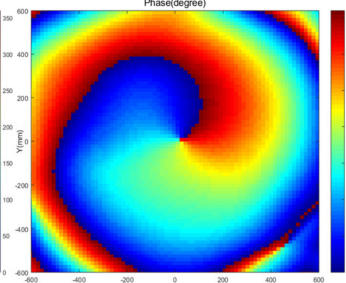

(f)

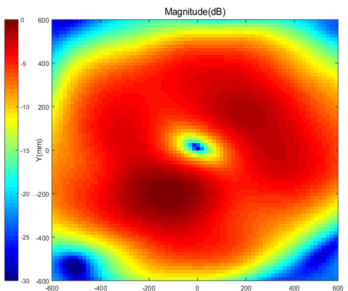

(c)

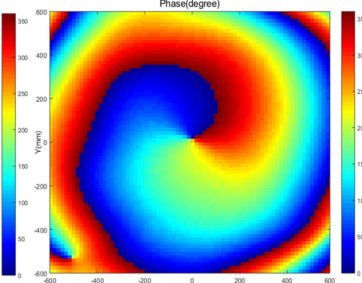

(g)

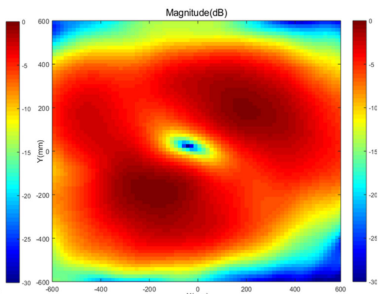

(d)

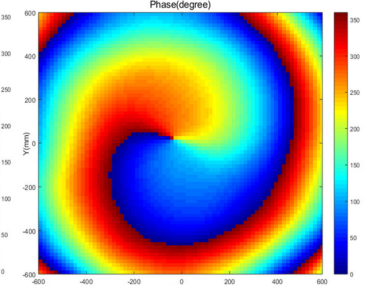

(h)

Figure 8. Measured near-field amplitude and phase distributions on the sampling plane vertical to the scanning beams approximately 25 wavelengths away from the reconfigurable surface. Amplitude distributions of OAM scanning beams with the mode $l=1$ at (a) $15^{\circ}$, (b) $30^{\circ}$, (c) $45^{\circ}$, and (d) $60^{\circ}$. Phase distributions of OAM scanning beams with the mode $l=1$ at (e) $15^{\circ}$, (f) $30^{\circ}$, (g) $45^{\circ}$, and (h) $60^{\circ}$.

The OAM beam scanning has been realized in a fixed azimuth plane. Furthermore, the beam can be scanned in different azimuth planes. As shown in Figure 9a,b the OAM beam has achieved scanning from $\varphi=0^{\circ}$ to $\varphi=270^{\circ}$ with a fixed $\theta=15^{\circ}$. In addition, 3D patterns are presented to show that the beam changes more vividly in Figure 9c,d. Therefore, it is satisfactory for the scanning ability of an OAM beam in arbitrary azimuth planes. In summary, the real-time control for beam scanning of OAM in the 3D domain is achieved. 

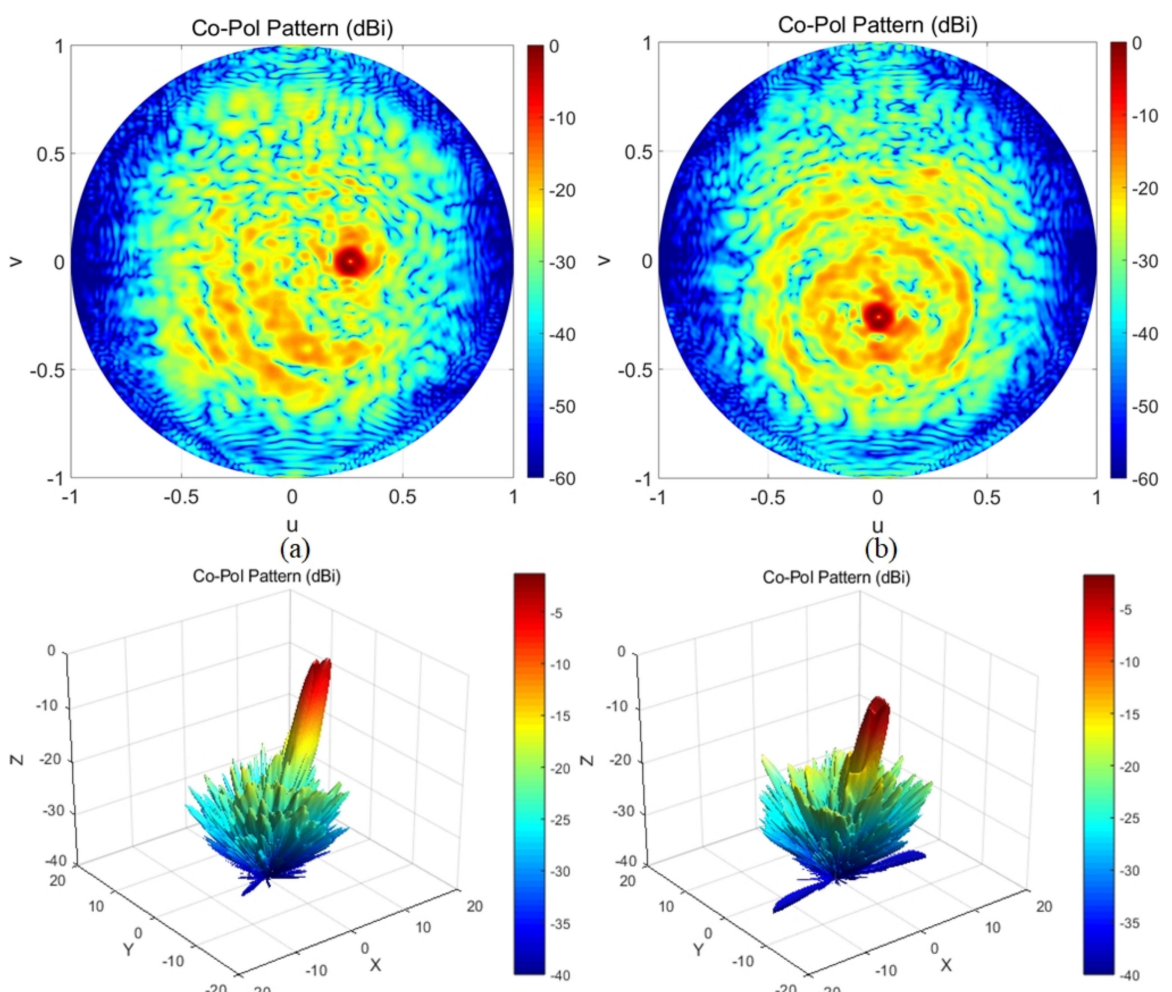

(c)

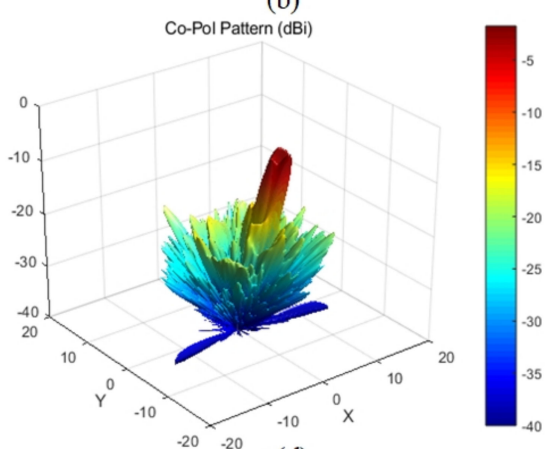

Figure 9. Measured far-field patterns with different azimuth directions in different coordinate systems: UV: (a) $\varphi=0^{\circ}$; (b) $\varphi=270^{\circ} ;$ polar (c) $\varphi=0^{\circ} ;$ (d) $\varphi=270^{\circ}$.

\subsection{Performance Comparison}

Table 2 presents some classical OAM-generating antennas for OAM mode switching based on different methods compared with this proposed 1-bit reconfigurable reflectarray antenna. It is clear that either feed or the radiation surface is reconfigurable for the adjustable function of OAM beams. Furthermore, the OAM mode switching is realized by using a reconfigurable feed network in [17], and a multiple-polarization OAM beam [24] can be realized and switches between each other by rotating the linear-polarization feed. Both of the above-mentioned antennas $[17,24]$ for adjustable OAM beams are realized by adjusting the feed. Thereafter, the reflective/transmissive reconfigurable antennas [36,38] are utilized to realize the OAM switching by modifying the phase encoding sequence on the surface. It is noteworthy that the proposed reflectarray surface has the advantages of high gain and large scanning angle comparing with other reference antennas.

Table 2. Comparison with other published OAM antennas.

\begin{tabular}{ccccccc}
\hline Ref & Type & $\begin{array}{c}\text { Frequency } \\
(\mathbf{G H z})\end{array}$ & OAM Mode & Mode Switching & $\begin{array}{c}\text { OAM Beam } \\
\text { Scanning }\end{array}$ & Gain(dBi) \\
\hline$[17]$ & UCA & 2.5 & $+1 /-1$ & Yes(CP + Mode) & No & 5.3 \\
\hline$[24]$ & RA & 10 & +1 & Yes (CP/LP) & No & Not Given \\
\hline$[36]$ & RRA & 4.75 & $+1 /+2$ & Yes & No & Not Given \\
\hline$[38]$ & RTA & 7.5 & $\pm 1 / \pm 2 / 0$ & Yes & No & Not Given \\
\hline This Paper & RRA & 9.37 & $0 / \pm 1$ & Yes & Yes & 27.2 \\
\hline
\end{tabular}

Remarks: Uniform Circular Array (UCA), Reflective Array (RA), Transmission Array (TA), Reconfigure Reflective Array (RRA), Reconfigure Transmission Array (RTA). 


\section{Conclusions}

A 1-bit reconfigurable reflectarray surface containing 2304 individually controlled elements has been utilized to present the real-time dynamic control of OAM beams. First, the phase distributions for different OAM beams using 1-bit element are presented. Then, the real-time control of OAM mode switching between $l=1$ and $l=2$ is realized by revising the phase states of PIN diodes on the reconfigurable surface using FPGA. Next, the beam scanning of OAM is conveniently obtained as expected, and a large scanning angle of the OAM wave at $75^{\circ}$ was also achieved. In addition, it is satisfactory for the scanning ability of an OAM beam in arbitrary azimuth planes.

The real-time dynamical control of OAM mode switching and large-angle beam scanning in 3D space are both achieved. Compared to the traditional approaches for OAM generation, it is flexible for high-gain OAM mode switching by using the 1-bit reconfigurable surface. Meanwhile, the capability of the large-scale reconfigurable surface is powerful, and other variable high-order modes of OAM such as $l=3$ or $l=5$ can also be realized. Moreover, the range of OAM beam scanning is wide, and the OAM beam direction is accurate and controllable with high gain. Therefore, the results suggest that it can satisfy the requirements of real-time dynamic communication systems in the future.

Author Contributions: Conceptualization, Z.W. and F.Y.; methodology, Z.W. and F.Y.; software, Z.W. and X.P.; validation, Z.W., X.P., S.X., and M.L.; formal analysis, M.L.; investigation, Z.W.; resources, Z.W.; data curation, S.X.; writing —original draft preparation, Z.W.; writing—review and editing, Z.W.; visualization, Z.W.; supervision, F.Y.; project administration, Z.W.; funding acquisition, Z.W. and F.Y. All authors have read and agreed to the published version of the manuscript.

Funding: This research received no external funding.

Acknowledgments: Project funded by China Postdoctoral Science Foundation (2019M650677).

Conflicts of Interest: The authors declare no conflict of interest.

\section{References}

1. Thidé, B.; Tamburini, F.; Then, H.; Someda, C.G.; Ravanelli, R.A. The physics of angular momentum raido. In Proceedings of the 1st URSI Atlantic Radio Science Conference, Gran Canaria, Spain, 16-24 May 2015.

2. Poynting, J.H. On Pressure Perpendicular to the Shear Planes in Finite Pure Shears, and on the Lengthening of Loaded Wires When Twisted. Proc. R. Soc. A Math. Phys. Eng. Sci. 1909, 82, 546.

3. Allen, L.; Beijersbergen, M.W.; Spreeuw, R.J.C.; Woerdman, J. Orbital angular momentum of light and transformation of Laguerre Gaussian Laser modes. Phys. Rev. A 1992, 45, 8185. [CrossRef] [PubMed]

4. Bretschneider, S.; Eggeling, C.; Hell, S.W. Breaking the Diffraction Barrier in Fluorescence Microscopy by Optical Shelving. Phys. Rev. Lett. 2007, 98, 218103. [CrossRef] [PubMed]

5. Zheng, S.L.; Hui, X.N.; Zhu, J.B.; Chi, H.; Jin, X.F.; Yu, S.Y.; Zhang, X.M. Orbital angular momentum mode-demultiplexing scheme with partial angular receiving aperture. Opt. Lett. 2015, 23, 12251. [CrossRef] [PubMed]

6. Franke, S.; Allen, L.; Padgett, M. Advances in optical angular momentum. Laser Photonics Rev. 2010, 2, 299. [CrossRef]

7. Chen, M.Z.; Michael, M.; Arita, Y.; Wright, E.; Dholakia, K. Dynamics of microparticles trapped in a perfect vortex beam. Opt. Lett. 2013, 15, 4919. [CrossRef]

8. Grier, D.G. A revolution in optical manipulation. Nature 2003, 424, 810. [CrossRef]

9. Skarka, V.; Aleksi, N.B.; Berezhiani, V.I. Evolution of singular optical pulses towards vortex solitons and filamentation in air. Phys. Lett. A 2003, 319, 317. [CrossRef]

10. Oemrawsingh, S.S.R.; Aiello, A.; Eliel, E.R.; Nienhuis, G.; Woerdman, J.P. How to Observe High-Dimensional Two-Photon Entanglement with Only Two Detectors. Phys. Rev. Lett. 2004, 92, 217901. [CrossRef]

11. Thidé, B.; Then, H.; Sjöholm, J.; Palmer, K.; Bergman, J.; Carozzi, T.D.; Istomin, Y.N.; Ibragimov, N.H.; Khamitova, R. Utilization of Photon Orbital Angular Momentum in the Low-Frequency Radio Domain. Phys. Rev. Lett. 2007, 99, 087701.

12. Tamburini, F.; Mari, E.; Thidé, B.; Barbieri, C.; Romanato, F. Experimental verification of photon angular momentum and vorticity with radio techniques. Appl. Phys. Lett. 2011, 99, 204102. [CrossRef] 
13. Tamburini, F.; Mari, E.; Sponselli, A.; Thidé, B.; Bianchini, A.; Romanato, F. Encoding many channels in the same frequency through radio vorticity: First experimental test. New J. Phys. 2012, 14, 33001. [CrossRef]

14. Niemiec, R.; Brousseau, C.; Mahdjoubi, K.; Emile, O.; Ménard, A. Characterization of an OAM Flat Plate Antenna in the Millimeter Frequency Band. IEEE Antennas Wirel. Propag. Lett. 2014, 13, 1011. [CrossRef]

15. Zhang, W.; Zheng, S.L.; Hui, X.N.; Chen, Y.L.; Jin, X.F.; Chi, H.; Zhang, X.M. Four-OAM-mode Antenna with Traveling-wave Ring-slot Structure. IEEE Antennas Wirel. Propag. Lett. 2016, 16, 194. [CrossRef]

16. Liu, B.Y.; Cui, Y.H.; Li, R.L. A Broadband Dual-Polarized Dual-OAM-Mode Antenna Array for OAM Communication. IEEE Antennas Wirel. Propag. Lett. 2017, 16, 744. [CrossRef]

17. Liu, Q.; Chen, Z.N.; Liu, Y.; Li, F.; Chen, Y.; Mo, Z. Circular Polarization and Mode Reconfigurable Wideband Orbital Angular Momentum Patch Array Antenna. IEEE Trans. Antennas Propag. 2018, 66, 1796-1804. [CrossRef]

18. Ren, J.; Leung, K.W. Generation of microwave orbital angular momentum states using hemispherical dielectric resonator antenna. Appl. Phys. Lett. 2018, 112, 131103. [CrossRef]

19. Yang, Y.; Wang, Z.; Wang, S.; Zhou, Q.; Shen, F.; Jiang, H.; Wu, Z.; Zeng, B.; Guo, Z.; Gong, Y. Designing a Water-Immersed Rectangular Horn Antenna for Generating Underwater OAM Waves. Electronics 2019, 8, 1224. [CrossRef]

20. Wang, L.; Chen, H.; Guo, K.; Shen, F.; Guo, Z. An Inner- and Outer-Fed Dual-Arm Archimedean Spiral Antenna for Generating Multiple Orbital Angular Momentum Modes. Electronics 2019, 8, 251. [CrossRef]

21. Yu, S.X.; Li, L.; Shi, G.M.; Zhu, C.; Zhou, X.X.; Shi, Y. Design, fabrication, and measurement of reflective metasurface for orbital angular momentum vortex wave in radio frequency domain. Appl. Phys. Lett. 2016, 108, 121903. [CrossRef]

22. Yu, S.X.; Li, L.; Shi, G. Dual-polarization and dual-mode orbital angular momentum radio vortex beam generated by using reflective metasurface. Appl. Phys. Lett. 2016, 9, 082202. [CrossRef]

23. Meng, X.S.; Wu, J.J.; Wu, Z.S.; Qu, T.; Yang, L. Dual-polarized reflectarray for generating dual beams with two different orbital angular momentum modes based on independent feeds in C-and X-bands. Opt. Express 2018, 26, 23185. [CrossRef] [PubMed]

24. Meng, X.S.; Wu, J.J.; Wu, Z.S.; Qu, T.; Yang, L. Design of multiple-polarization reflectarray for orbital angular momentum wave in radio frequency. IEEE Antennas Wirel. Propag. Lett. 2018, 17, 2269. [CrossRef]

25. Yu, S.; Li, L.; Shi, G.; Zhu, C.; Shi, Y. Generating multiple orbital angular momentum vortex beams using a metasurface in radio frequency domain. Appl. Phys. Lett. 2016, 108, 241901. [CrossRef]

26. Akram, Z.; Li, X.P.; Qi, Z.H.; Aziz, A.; Jiang, X.; Li, X.M. Broadband High-order OAM Reflective Metasurface with High Mode Purity Using Subwavelength Element and Circular Aperture. IEEE Access 2019, 7, 71963. [CrossRef]

27. Jiang, S.; Chen, C.; Zhang, H.; Chen, W. Achromatic electromagnetic metasurface for generating a vortex wave with orbital angular momentum (OAM). Opt. Express 2018, 26, 6466. [CrossRef] [PubMed]

28. Meng, X.S.; Wu, J.J.; Wu, Z.S.; Yang, L.; Huang, L.; Li, X.; Qu, T. Design, fabrication, and measurement of an anisotropic holographic metasurface for generating vortex beams carrying orbital angular momentum. Opt. Lett. 2019, 44, 1452. [CrossRef]

29. Yang, J.; Zhang, C.; Ma, H.F.; Zhao, J.; Dai, J.Y.; Yuan, W.; Yang, L.X.; Cheng, Q.; Cui, T.J. Generation of radio vortex beams with designable polarization using anisotropic frequency selective surface. Appl. Phys. Lett. 2018, 112, 203501. [CrossRef]

30. Yang, H.; Yang, F.; Xu, S.H.; Li, M.K.; Cao, X.Y.; Gao, J. A 1-Bit Multi-Polarization Reflectarray Element for Reconfigurable Large Aperture Antennas. IEEE Antennas Wirel. Propag. Lett. 2017, 16, 581. [CrossRef]

31. Yang, H.; Yang, F.; Cao, X.; Xu, S.; Gao, J.; Chen, X.; Li, M.; Li, T. A 1600-Element Dual-Frequency Electronically Reconfigurable Reflectarray at X/Ku Bands. IEEE Trans. Antennas Propag. 2017, 65, 3024. [CrossRef]

32. Wang, Y.; Xu, S.H.; Yang, F.; Werner, D.H. 1-Bit Dual-Linear Polarized Reconfigurable Transmitarray Antenna Using Asymmetric Dipole Elements with Parasitic Bypass Dipoles. IEEE Trans. Antennas Propag. 2020, early access.

33. Nguyen, B.D.; Pichot, C. Unit-Cell Loaded with PIN Diodes for 1-Bit Linearly Polarized Reconfigurable Transmittarrays. IEEE Antennas Wirel. Propag. Lett. 2019, 18, 98-102. [CrossRef]

34. Palma, L.D.; Clemente, A.; Dussopt, L.; Sauleau, R.; Potier, P.; Pouliguen, P. 1-Bit Reconfigurable Unit Cell for ka-Band Transmitarrays. IEEE Antennas Wirel. Propag. Lett. 2016, 15, 560. [CrossRef] 
35. Huang, C.; Pan, W.; Ma, X.; Zhao, B.; Cui, J.; Luo, X. Using Reconfigurable Transmitarray to Achieve Beam-Steering and Polarization Manipulation Applications. IEEE Trans. Antennas Propag. 2015, 63, 4801. [CrossRef]

36. Han, J.Q.; Li, L.; Yi, H.; Shi, Y. 1-bit digital orbit angular momentum vortex beam generator based on a coding reflective metasurface. Opt. Mater. Express 2018, 8, 3470. [CrossRef]

37. Han, J.Q.; Li, L.; Yi, H.; Xue, W.M. Versatile orbital angular momentum vortex beam generator based on reconfigurable reflective metasurface. Jpn. J. Appl. Phys. 2018, 57, 120303. [CrossRef]

38. Bai, X.D.; Kong, F.W.; Sun, Y.T.; Wang, G.F.; Qian, J.Y.; Li, X.B.; Cao, A.J.; He, C.; Liang, X.L.; Jin, R.H.; et al. High efficiency Transmissive Programmable Metasurface for Multimode OAM Generation. Adv. Opt. Mater. 2020, 45, 2000570. [CrossRef]

Publisher's Note: MDPI stays neutral with regard to jurisdictional claims in published maps and institutional affiliations.

(C) 2020 by the authors. Licensee MDPI, Basel, Switzerland. This article is an open access article distributed under the terms and conditions of the Creative Commons Attribution (CC BY) license (http://creativecommons.org/licenses/by/4.0/). 